\title{
Improving SVD-based image watermarking via block-by-block optimization on singular values

\author{
Huang-Nan Huang ${ }^{1}$, Der-Fa Chen², Chiu-Chun Lin², Shuo-Tsung Chen ${ }^{1 *}$ and Wei-Che Hsu ${ }^{3}$
}

\begin{abstract}
The purpose of this paper is to improve the robustness of traditional image watermarking based on singular value decomposition (SVD) by using optimization-based quantization on multiple singular values in the wavelet domain. In this work, we divide the middle-frequency parts of discrete-time wavelet transform (DWT) into several square blocks and then use multiple singular value quantizations to embed a watermark bit. To minimize the difference between original and watermarked singular values, an optimized-quality formula is proposed. First, the peak signal-to-noise ratio (PSNR) is defined as a performance index in a matrix form. Then, an optimized-quality functional that relates the performance index to the quantization technique is obtained. Finally, the Lagrange Principle is utilized to obtain the optimized-quality formula and then the formula is applied to watermarking. Experimental results show that the watermarked image can keep a high PSNR and achieve better bit-error rate (BER) even when the number of coefficients for embedding a watermark bit increases.
\end{abstract}

\section{Introduction}

With the rapid development of activity on the internet, much digital information is widely spread. Digital watermarking was developed to hide digital information and protect the copyright of multimedia signals, like audio, images, etc. Due to the fact that discrete-time wavelet transform (DWT) provides a useful platform, numerous DWT-based algorithms for digital watermarking have been proposed in recent years.

Watermarking in the spatial domain [1-11] is usually more vulnerable than watermarking in the frequency domain [12-29] with the same embedding capacity due to the fact that spatial-domain methods are generally fragile to image-processing operations and other attacks [23-25]. The spatial-domain singular value decomposition (SVD) for image watermarking was first introduced by Liu et al. [8]. In this paper, the authors used a spread-spectrum technique to embed a watermark by modifying the singular values of the host image in the spatial domain. Some authors embedded watermark to $U$ and $V$ components to increase embedding capacity $[9,10]$ while Ghazy et al. [11] presented a blockby-block SVD-based image-watermarking scheme to

\footnotetext{
* Correspondence: shough34@yahoo.com.tw

'Department of Applied Mathematics, Tunghai University, Taichung 407, Taiwan Full list of author information is available at the end of the article
}

increase embedding capacity. However, the robustness of SVD-based image watermarking in the spatial domain is low. In recent years, many image-watermarking techniques combine DWT and SVD to achieve better transparency and robustness [17, 18, 24, 25]. Bao et al. [17] proposed a novel, yet simple, image-adaptive watermarking scheme for image authentication by applying a simple quantizationindex-modulation process on each single singular value of the blocks in the wavelet domain. Their watermarking scheme is blind and is robust against JPEG compression but extremely sensitive to malicious manipulation such as filtering and random noising. Ganic et al. [18] applied SVD to all details, approximating part of the DWT and watermark image to increase embedding capacity. Gaurav and Balasubramanian [24] embedded a watermark into the reference image by modifying the singular value of the reference image using the singular values of the watermark. The robustness is slightly enhanced. However, the computation is significantly increased. Lai and Tsai [25] reduced the computation in [24] by directly embedding the watermark into the singular values in the wavelet domain.

In this work, we first divide the DWT middlefrequency parts LH3 and HL3 into several square blocks to have high embedding capacity. Unlike the traditional spread-spectrum technique on single singular values $[24,25]$, we use multiple singular value quantizations to embed a watermark bit. It does not only keep a high

\section{Springer}


embedding capacity but also achieves strong robustness against median filtering. On the other hand, an optimizedquality formula is proposed by minimizing the difference between original and watermarked singular values. First, the peak signal-to-noise ratio (PSNR) is defined as a performance index in matrix form. Then, an optimizedquality functional that relates the performance index to the quantization technique is obtained. Finally, the Lagrange Principle is utilized to obtain the optimizedquality formula; then, the formula is applied to watermarking. Experimental results show that the watermarked image can keep a high PSNR and achieve a better biterror rate (BER) even when the number of coefficients for embedding a watermark bit increases. In particular, the robustness against median filtering is significantly improved.

This paper is organized as follows. In Section II, we review some mathematical preliminaries. Section III introduces the proposed watermark embedding and extraction. In Section IV, we rewrite PSNR as a performance index. An optimized-quality equation that relates the performance index to the quantization constraint is proposed, and the Lagrange Principle is used to solve the optimized-quality problem. The solution is utilized to embed the watermark, and we discover a very good result; the watermark is extracted without the original image. In Section V, we present some experiments to test the performance of the proposed scheme. Finally, conclusions are drawn in Section VI.

\section{Preliminaries}

In this section, some related steps for the proposed imagewatermarking scheme are reviewed.

\subsection{Discrete-time wavelet transform (DWT)}

The wavelet transform is obtained by a single prototype function which is regulated with a scaling parameter and shift parameter [28-31]. The discrete normalized scaling and wavelet basis function are defined as follows:

$$
\begin{aligned}
& \varphi_{j, l}(t)=2^{j / 2} \varphi\left(2^{j} t-\tau\right) \\
& \psi_{j, l}(t)=2^{j / 2} \psi\left(2^{j} t-\tau\right)
\end{aligned}
$$

where $j$ and $\tau$ are the dilation and translation parameters; from this, one can require that the sequence

$$
\{0\} \subset \cdots \subset V_{1} \subset V_{0} \subset V_{-1} \subset \cdots \subset L^{2}(\mathbb{R})
$$

forms a mutiresolution analysis of $L^{2}(\mathbb{R})$ and that the subspaces ..., $W_{1}, W_{0}, W_{-1}, \ldots$ are the orthogonal differences of the above sequence; that is, $W_{j}$ is the orthogonal complement of $V_{j}$ inside the subspace $V_{j-1}$. Then, the orthogonality relations follow from the existence of sequences $h=\left\{h_{\tau}\right\}_{\tau \in Z}$ and $g=\left\{g_{\tau}\right\}_{\tau \in Z}$ that satisfy the following identities:

$$
\begin{aligned}
& h_{\tau}=\varphi_{0,0}, \varphi_{-1, \tau} \text { and } \varphi(t)=\sqrt{2} \sum_{\tau \in \mathbb{Z}} h_{\tau} \varphi(2 t-\tau) \\
& g_{\tau}=\psi_{0,0}, \phi_{-1, \tau} \text { and } \psi(t)=\sqrt{2} \sum_{\tau \in \mathbb{Z}} g_{\tau} \varphi(2 t-\tau)
\end{aligned}
$$

where $h=\left\{h_{\tau}\right\}_{\tau \in Z}$ and $g=\left\{g_{\tau}\right\}_{\tau \in Z}$ are, respectively, the sequence of low-pass and high-pass filters. In this paper, we use a Haar scaling function and wavelet to transform the host image into the orthogonal DWT domain by three-level decomposition. A method to implement DWT is a filter bank that provides perfect reconstruction. DWT has local analysis of frequency in the space and time domains, and it obtains image multi-scale details step by step. If the scale becomes smaller, every part gets more accurate and ultimately all image details can be focalized accurately. If DWT is applied to an image, it will produce high-frequency parts, middle-frequency parts, and a lowest-frequency part. Figure 1 shows the procedure of applying one-level DWT to an image. In order to guarantee both image quality and robustness, this study embeds the watermark into the middlefrequency parts LH3 and HL3 in DWT level-three.

\subsection{Singular value decomposition (SVD)}

The singular value decomposition of a matrix $A$ with size $m \times n$ is given by

$$
A=U D V^{T}
$$

where $U$ and $V$ are orthogonal matrices, and $D=\operatorname{diag}\left(\lambda_{i}\right)$ is a diagonal matrix of singular values $\lambda_{i}, i=1,2, \cdots$, which are arranged in decreasing order. The columns of $U$ are the left singular vectors, and the columns of $V$ are the right singular vectors of image $A$.

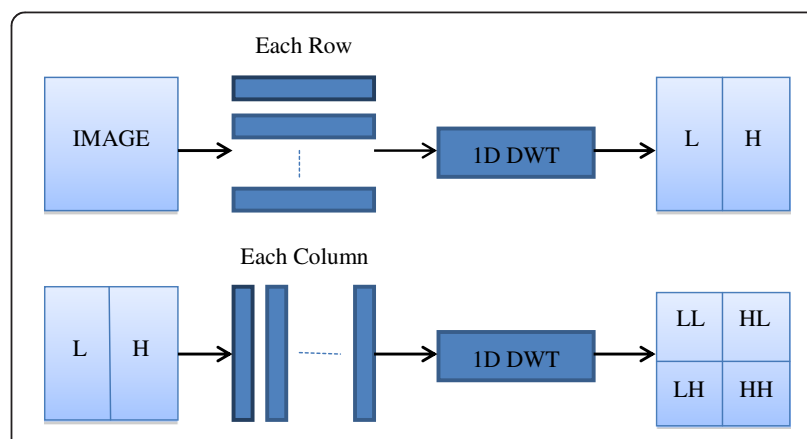

Fig. 1 2D DWT 


\subsection{Optimization solver}

To find the extreme of the matrix function, some optimization methods are summarized in [29-31]. The operations of the matrix function are first shown as follows.

Theorem 1. If $\mathbf{W}$ is a $k \times k$ constant matrix, and $\widehat{\mathrm{X}}$ is $a k \times 1$ column vector with $k$ unknown variables, then

$$
\frac{\partial \mathbf{W} \widehat{\mathrm{X}}}{\partial \widehat{\mathrm{X}}}=\mathbf{W}
$$

Theorem 2. If $\mathrm{X}$ is a $k \times 1$ constant vector and $\widehat{\mathrm{X}}$ is a $k \times 1$ column vector with $k$ unknown variables, then

$$
\frac{\partial(\widehat{X}-X)^{T}(\widehat{X}-X)}{\partial(\widehat{X}-X)}=2(\widehat{X}-X)
$$

In order to apply the Lagrange Principle, we have to introduce the gradient of a matrix function $f(\overline{\mathbf{C}})$ as follows.

Definition 1. Suppose that $\widehat{\mathrm{X}}=\left[\hat{x}_{1} \hat{x}_{2} \ldots \hat{x}_{k}\right]^{T}$ is a $k \times 1$ unknown vector and $f(\widehat{\mathrm{X}})$ is a function of the vector $\widehat{\mathrm{X}}$, the gradient of $f(\widehat{\mathrm{X}})$ is

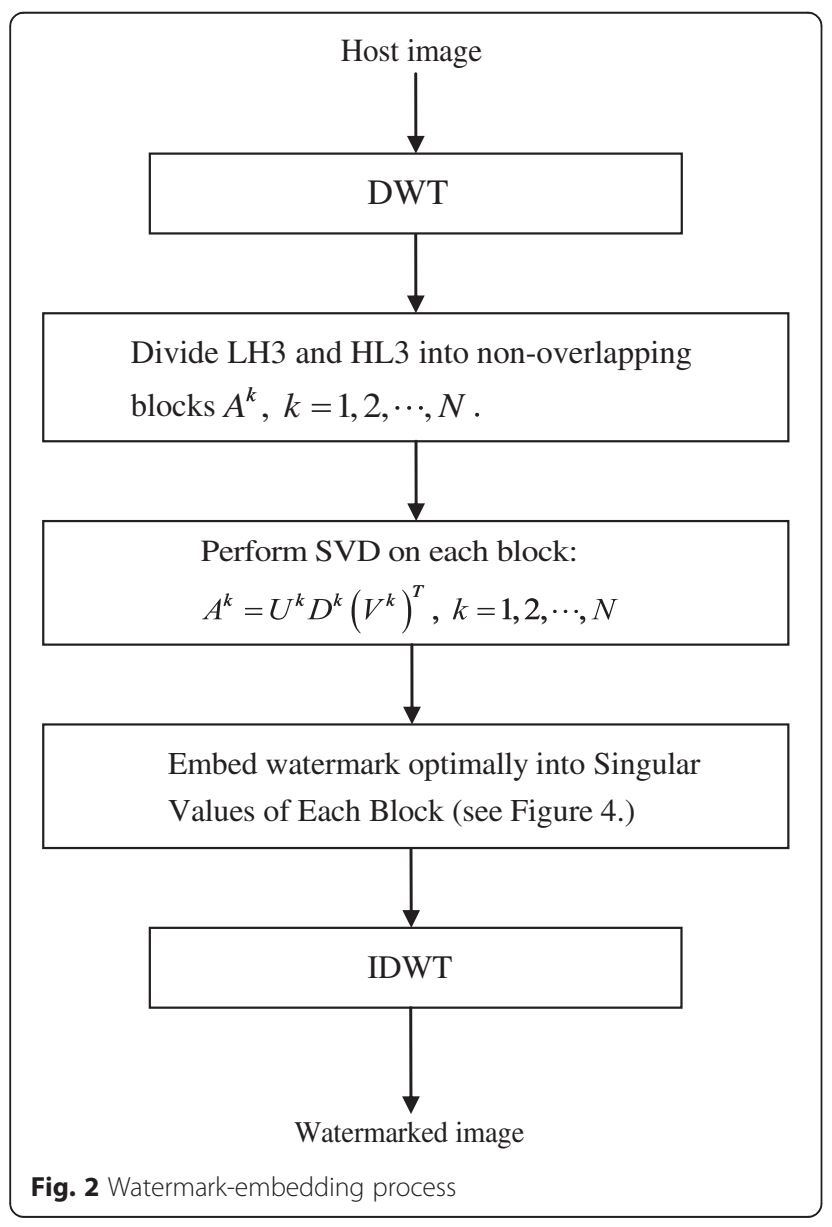

$$
\nabla f(\widehat{\mathrm{X}})=\frac{\partial f}{\partial \widehat{\mathrm{X}}}=\left[\frac{\partial f}{\partial \widehat{\mathrm{X}}_{1}} \frac{\partial f}{\partial \widehat{\mathrm{X}}_{2}} \cdots \frac{\partial f}{\partial \widehat{\mathrm{X}}_{k}}\right]^{T}
$$

Now we consider the problem of minimizing (or maximizing) the matrix function $f(\widehat{\mathrm{X}})$ subject to a constraint $(\widehat{\mathrm{X}})=0$. This problem can be described as follows:

$$
\begin{aligned}
& \text { minimize } f(\widehat{\mathrm{X}}) \\
& \text { subject to } \mathrm{g}(\widehat{\mathrm{X}})=0
\end{aligned}
$$

In order to solve (9), we apply the Lagrange Principle as follows.

Theorem 3. Suppose that $g$ is a continuously differentiable function of $\widehat{\mathrm{X}}$ on a subset of the domain of a function $f$. Then if $\widehat{\mathrm{X}}_{0}$ minimizes (or maximizes) $f(\widehat{\mathrm{X}})$ subject to the constraint $(\widehat{\mathrm{X}})=0, \nabla f\left(\widehat{\mathrm{X}}_{0}\right)$ and $\nabla g\left(\widehat{\mathrm{X}}_{0}\right)$ are parallel. That is, if $\left(\widehat{\mathrm{X}}_{0}\right) \neq 0$, then there exists a scalar $\xi$ such that

$$
\nabla f\left(\widehat{\mathrm{X}}_{0}\right)=\xi \nabla g\left(\widehat{\mathrm{X}}_{0}\right)
$$

Based on Theorem 3, if we let

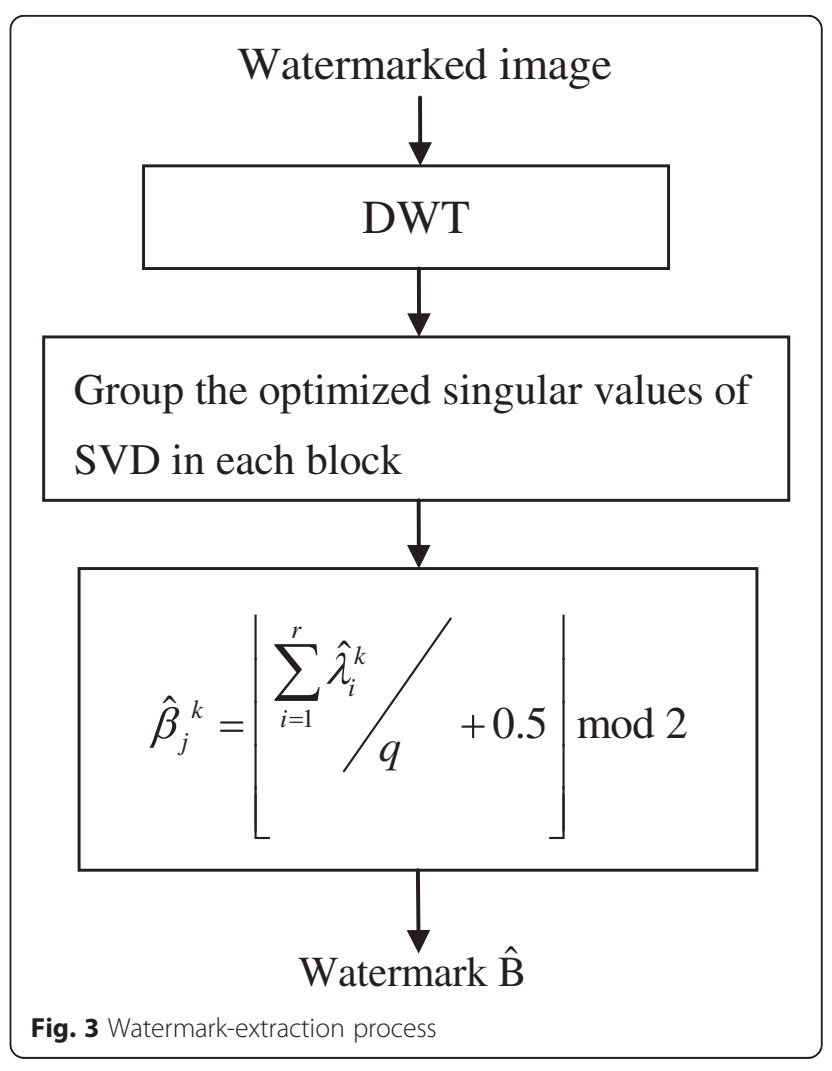




$$
H(\widehat{\mathrm{X}}, \xi)=f(\widehat{\mathrm{X}})+\xi g(\widehat{\mathrm{X}})
$$

then the original problem (9) becomes a function $H$ $(\widehat{\mathrm{X}}, \xi)$ which has no constraint. The necessary conditions for existence of the extreme of $H(\widehat{\mathrm{X}}, \xi)$ are

$$
\begin{aligned}
& \frac{\partial H}{\partial \xi}=0, \\
& \frac{\partial H}{\partial \widehat{\mathrm{X}}}=0 .
\end{aligned}
$$

\section{Proposed optimization-based DWT-SVD watermarking scheme}

The proposed watermarking scheme is introduced in this section. The watermark is extracted without the original image.

\subsection{Watermark embedding}

The proposed embedding process as shown in Fig. 2 is summarized as follows.

(1) Use three-level DWT to decompose the original image A into four subbands (i.e., LL3, LH3, HL3, and HH3).

(2)Divide LH3 and HL3 into non-overlapping blocks $A^{k}, k=1,2, \cdots, N$.

(3) Apply SVD to each block, i.e.,

$$
A^{k}=U^{k} D^{k}\left(V^{k}\right)^{T}, \quad k=1,2, \cdots, N
$$

where $k$ represents the number of blocks in LH3 and HL3.

(4) Watermark $B=\left\{\beta_{j}\right\}$ randomly generated using a binary $\mathrm{PN}$ sequence is embedded by modifying

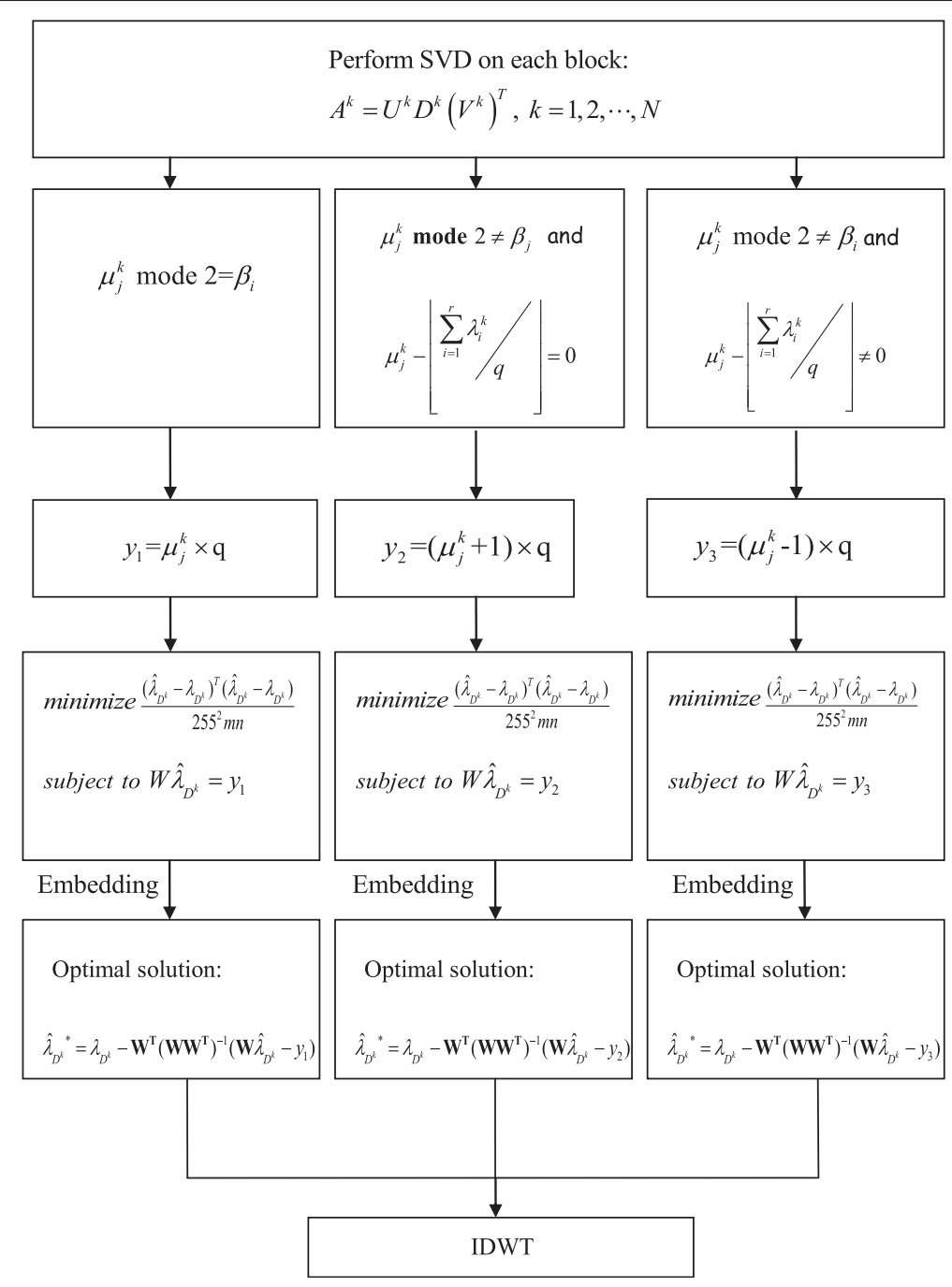

Fig. 4 Optimal embedding 
singular values $\lambda_{i}^{k}, i=1, \cdots, r$ of the matrix $A^{k}$ as follows: Let

$$
\mu_{j}^{k}=\left\lfloor\sum_{i=1}^{r} \lambda_{i}^{k} / q+\frac{1}{2}\right\rfloor,
$$

where $L\lrcorner$ indicates the floor function, and $q \in \mathbb{R}^{+}$is a quantization size which is adopted as another secret key $K_{1}$. The embedding rules have three situations as follows:

- If $\mu_{j}^{k}$ mode $2=\beta_{j}$, the singular values are modified to

$$
y_{1}=\mu_{j}^{k} \times q
$$

- If $\mu_{j}^{k}$ mode $2 \neq \beta_{j}$ and $\mu_{j}^{k}{ }_{-}\left\lfloor\sum_{i=1}^{r} \lambda_{i}^{k} /_{q}\right\rfloor=0$, the singular values are modified to

$$
y_{2}=\left(\mu_{j}^{k}+1\right) \times q
$$

- If $\mu_{j}^{k}$ mode $2 \neq \beta_{i}$ and $\mu_{j}^{k}-\left\lfloor\sum_{i=1}^{r} \lambda_{i}^{k} /_{q}\right\rfloor \neq 0$, the singular values are modified to

$$
y_{3}=\left(\mu_{j}^{k}-1\right) \times q
$$

\subsection{Watermark extraction}

To detect the watermark, DWT is first performed and then the optimized singular values of SVD in each block are grouped. The embedded binary bits are extracted by using the following rule.

$$
\hat{\beta}_{j}^{k}=\left\lfloor\sum_{i=1}^{r} \hat{\lambda}_{i}^{k} / q+0.5\right\rfloor \bmod 2,
$$

where $\hat{\beta}_{i}{ }^{k}$ denotes the extracted value and $\hat{\lambda}_{i}{ }^{k}$ are the singular values which are optimized by the proposed optimization in the next section. The hidden watermark bits (binary bits) are extracted as $B=\left\{\hat{\beta}_{i}^{k}\right\}$ without the original image. In other words, the proposed scheme is a blind watermarking scheme. Figure 3 shows the detailed process of the proposed watermark extraction.

\section{Optimization of PSNR on singular values}

Generally, the quality of a watermarked image is evaluated by the peak signal-to-noise ratio (PSNR). Since a

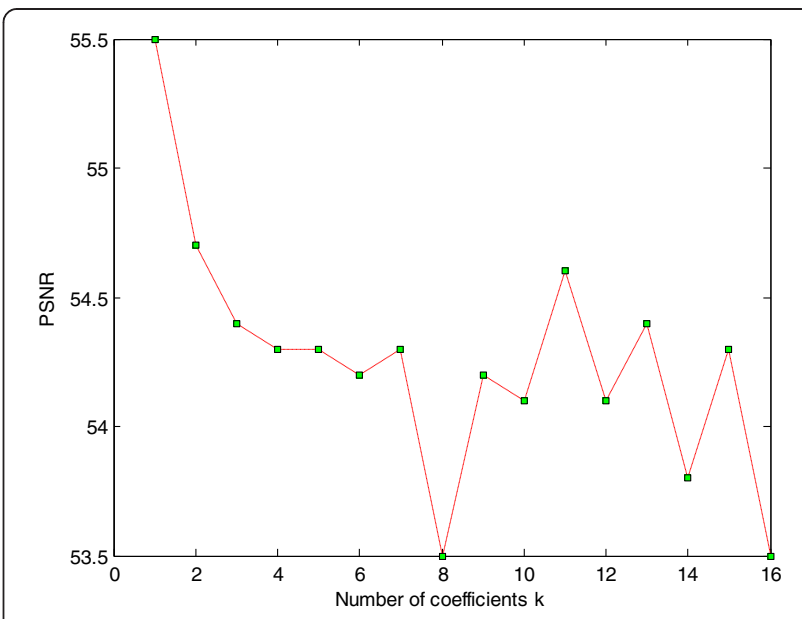

Fig. 5 The relation between PSNR and the number of coefficients

tradeoff exists between image quality measured by PSNR and robustness measured by BER, a scalar parameter $\xi$ is

\begin{tabular}{|c|c|c|c|}
\hline Method & Image genre & Parameters & $\begin{array}{l}\text { Embedding } \\
\text { capacity (bits) }\end{array}$ \\
\hline \multirow[t]{4}{*}{ Reference [24] } & Lena & $a=28$ & 256 \\
\hline & Jet & $a=25$ & 256 \\
\hline & Peppers & $a=28$ & 256 \\
\hline & Cameraman & $a=27$ & 256 \\
\hline \multirow[t]{4}{*}{ Reference [25] } & Lena & $a=28$ & 256 \\
\hline & Jet & $a=25$ & 256 \\
\hline & Peppers & $a=28$ & 256 \\
\hline & Cameraman & $a=27$ & 256 \\
\hline \multirow[t]{4}{*}{ Reference [27] } & Lena & $a=28$ & 256 \\
\hline & Jet & $a=25$ & 256 \\
\hline & Peppers & $a=28$ & 256 \\
\hline & Cameraman & $a=27$ & 256 \\
\hline \multirow[t]{12}{*}{ The proposed method } & Lena & $r=2, q=27$ & 512 \\
\hline & & $r=4, q=55$ & 256 \\
\hline & & $r=8, q=135$ & 64 \\
\hline & Jet & $r=2, q=26$ & 512 \\
\hline & & $r=4, q=44$ & 256 \\
\hline & & $r=8, q=129$ & 64 \\
\hline & Peppers & $r=2, q=28$ & 512 \\
\hline & & $r=4, q=51$ & 256 \\
\hline & & $r=8, q=131$ & 64 \\
\hline & Cameraman & $r=2, q=28$ & 512 \\
\hline & & $r=4, q=50$ & 256 \\
\hline & & $r=8, q=140$ & 64 \\
\hline
\end{tabular}
applied to connect the PSNR and the quantization equation to optimize the tradeoff in this section. The details are in the following:

Table 1 Embedding capacity under fixed PSNR 
First of all, the embedding technique can be rewritten as an equation according to Eqs. $(16,17,18)$ :

$$
W \hat{\lambda}_{D^{k}}=y_{1}, \text { if } " \mu_{j}^{k} \operatorname{mode} 2=\beta_{j} " .
$$

or

$$
W \hat{\lambda}_{D^{k}}=y_{2}, \text { if " } \mu_{j}^{k} \operatorname{mode} 2 \neq \beta_{i} \text { and } \mu_{j}^{k}{ }^{k}\left\lfloor\sum_{i=1}^{r} \lambda_{i}^{k} /_{q}\right\rfloor=0 " .
$$

or

$$
W \hat{\lambda}_{D^{k}}=y_{3}, \text { if " } \mu_{j}^{k} \operatorname{mode} 2 \neq \beta_{i} \text { and } \mu_{j}^{k}-\left\lfloor\sum_{i=1}^{r} \lambda_{i}^{k} /_{q}\right\rfloor \neq 0 " .
$$

where $W=\left[\begin{array}{lll}1 & \cdots & 1\end{array}\right]$ is a $1 \times r$ vector with all entries one, and the unknown vector $\hat{\lambda}_{D^{k}}=\left[\begin{array}{lll}\hat{\lambda}_{1}, & \cdots & \hat{\lambda}_{r}\end{array}\right]^{T}$ is the watermarked singular value vector that corresponds to $\lambda_{D^{k}}=\left[\begin{array}{lll}\lambda_{1}, & \cdots & \lambda_{r}\end{array}\right]^{T}$.

Next, we will connect the above quantization equations to the PSNR. If $\mathbf{I}(i, j)$ and $\mathbf{I}(i, j)$ are the values of the original and the corresponding modified pixel in the original image $\mathbf{I}$ and watermarked image $\overline{\mathbf{I}}$, then is defined as

$$
P S N R=-10 \log _{10}\left(\frac{\sum_{i=1}^{m} \sum_{j=1}^{n}(\mathbf{I}(i, j)-\mathbf{I}(i, j))^{2}}{255^{2} m n}\right),
$$

where $m$ and $n$ represent the height and width of the host image. Based on the watermark embedding of singular values, PSNR is expressed as

$$
\operatorname{PSNR}=-10 \log _{10}\left(\frac{\left\|\hat{\lambda}_{D^{k}}-\lambda_{D^{k}}\right\|_{2}^{2}}{255^{2} m n}\right)
$$

For the optimization of the watermarked image quality, Eq. 23 is rewritten as a performance index:

$$
f\left(\hat{\lambda}_{D^{k}}\right)=\frac{\left\|\hat{\lambda}_{D^{k}}-\lambda_{D^{k}}\right\|_{2}^{2}}{255^{2} m n}
$$

or

$$
f\left(\hat{\lambda}_{D^{k}}\right)=\frac{\left(\hat{\lambda}_{D^{k}}-\lambda_{D^{k}}\right)^{T}\left(\hat{\lambda}_{D^{k}}-\lambda_{D^{k}}\right)}{255^{2} m n}
$$

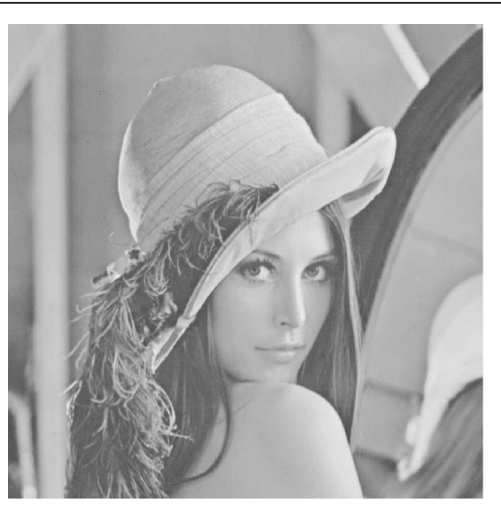

(a) Lena

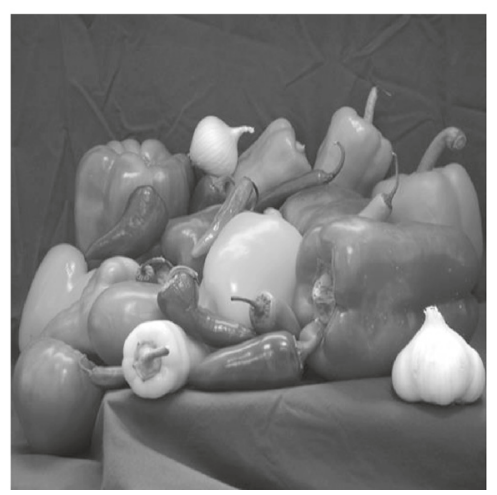

(c) Peppers

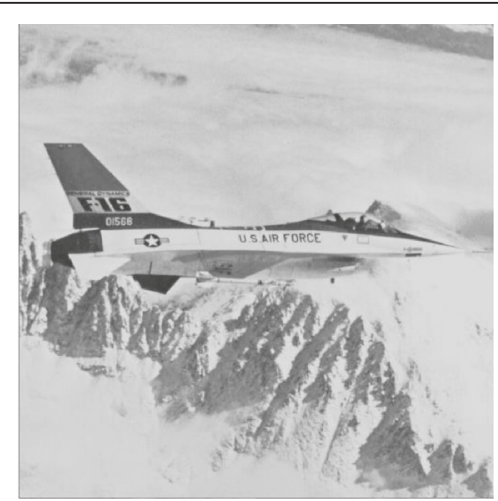

(b) Jet

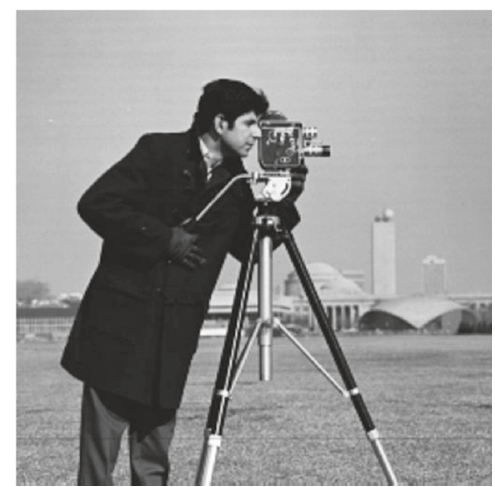

(d) Cameraman

Fig. 6 Original images. a Original image of Lena. b Original image of Jet. c Original image of Peppers. d Original image of Cameraman 
Based on the performance index $f\left(\hat{\lambda}_{D^{k}}\right)$ in Eq. 25 and the constraint in Eq. 19, when " $\mu_{j}^{k}$ mode $2=\beta_{i}$ ", the optimization-based quantization problem has the following form:

$$
\begin{aligned}
& \operatorname{minimize} \frac{\left(\hat{\lambda}_{D^{k}}-\lambda_{D^{k}}\right)^{T}\left(\hat{\lambda}_{D^{k}}-\lambda_{D^{k}}\right)}{255^{2} m n} \\
& \text { subject to } W \hat{\lambda}_{D^{k}}=y_{1}
\end{aligned}
$$

To embed the watermark $B$, we need to solve the optimization problem (26). By Theorem 3, we set the Lagrange multiplier $\lambda$ to combine (26a) and (26b) into a matrix function:

$$
H\left(\hat{\lambda}_{D^{k}}, \xi\right)=\frac{\left(\hat{\lambda}_{D^{k}}-\lambda_{D^{k}}\right)^{\mathbf{T}}\left(\hat{\lambda}_{D^{k}}-\lambda_{D^{k}}\right)}{255^{2} m n}+\xi\left(\mathrm{W} \hat{\lambda}_{D^{k}}-y_{1}\right)
$$

which has no constraint. Since $255^{2} m n$ is a constant, we redefine $H\left(\hat{\lambda}_{D^{k}}, \xi\right)$ as follows:

$$
\begin{aligned}
H\left(\hat{\lambda}_{D^{k}}, \xi\right)= & \left(\hat{\lambda}_{D^{k}}-\lambda_{D^{k}}\right)^{\top}\left(\hat{\lambda}_{D^{k}}-\lambda_{D^{k}}\right) \\
& +255^{2} m n \xi\left(\mathrm{W} \hat{\lambda}_{D^{k}}-y_{1}\right)
\end{aligned}
$$

The necessary conditions for existence of the minimum of $H\left(\hat{\lambda}_{D^{k}}, \xi\right)$ are

$$
\begin{aligned}
& \frac{\partial H}{\partial \hat{\lambda}_{D^{k}}}=2\left(\hat{\lambda}_{D^{k}}-\lambda_{D^{k}}\right)+255^{2} m n \xi \mathrm{W}^{\boldsymbol{\top}}=0 \\
& \frac{\partial H}{\partial \xi}=\mathrm{W} \hat{\lambda}_{D^{k}}-y_{1}=0
\end{aligned}
$$

Multiplying (29a) by W, we observe that

$$
2\left(\mathrm{~W} \hat{\lambda}_{D^{k}}-\mathrm{W} \lambda_{D^{k}}\right)+255^{2} m n \xi \mathrm{WW}^{\boldsymbol{\top}}=0
$$

Since $\mathrm{W} \hat{\lambda}_{D^{k}}=y_{1}$ from (29b) and $255^{2} m n$ is a scalar, we rewrite (30) as

$$
\left(y_{1}-\mathrm{W} \hat{\lambda}_{D^{k}}\right)+\frac{255^{2} m n}{2} \xi \mathrm{WW}^{\boldsymbol{\top}}=0
$$

Some operations yield the optimal solution for parameter $\xi$ as

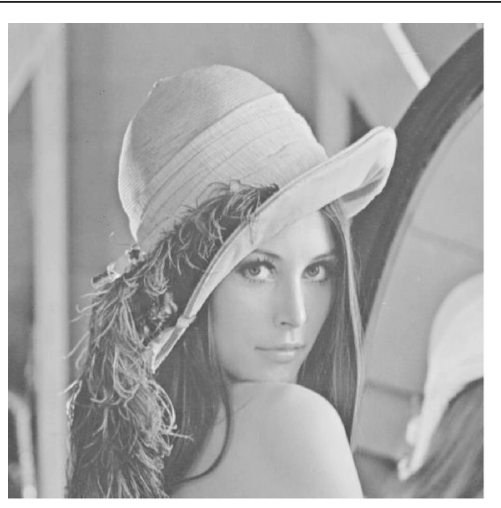

(a) Lena $(q=55)$

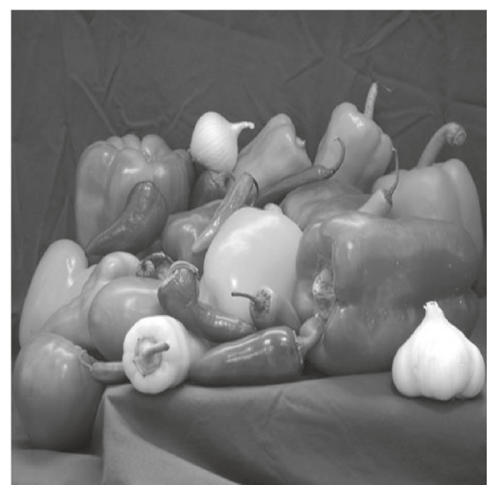

(c) Peppers $(q=51)$

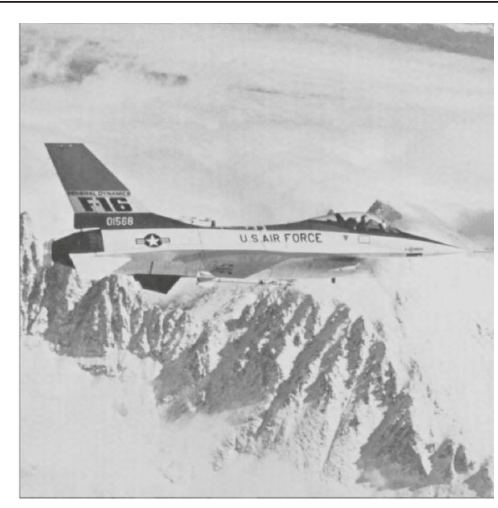

(b) Jet $(q=44)$

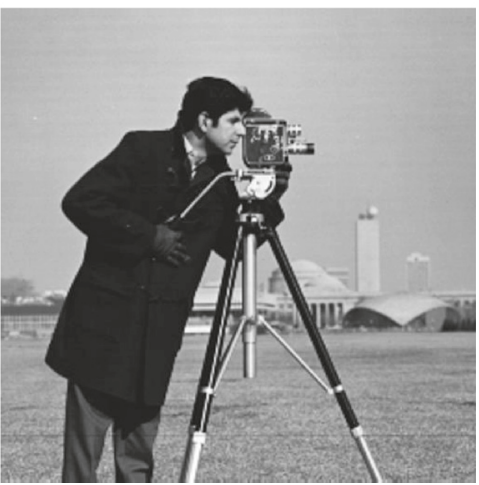

(d) Cameraman $(q=50)$

Fig. 7 Watermarked images for $k=4$. a Watermarked image of Lena $(q=55)$. $\mathbf{b}$ Watermarked image of Jet $(q=44)$. $\mathbf{c}$ Watermarked image of Peppers $(q=51)$. d Watermarked image of Cameraman $(q=50)$ 


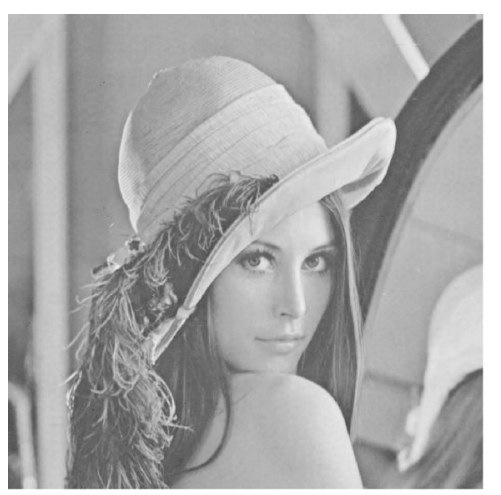

(a) Lena $(q=135)$

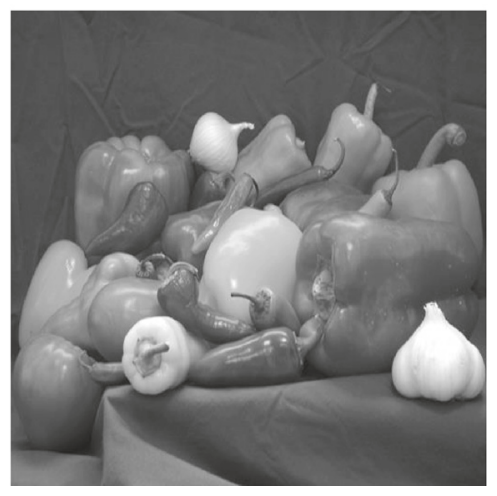

(c) Peppers $(q=131)$

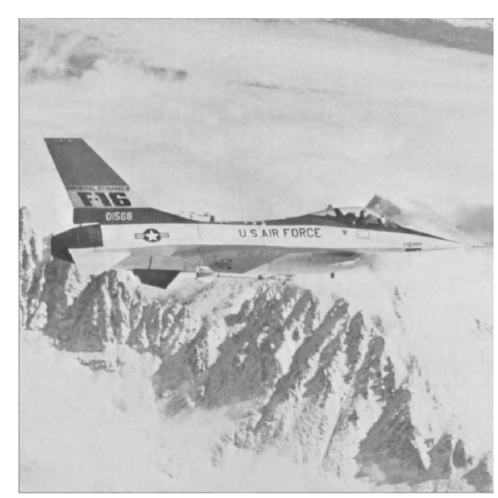

(b) Jet $(q=129)$

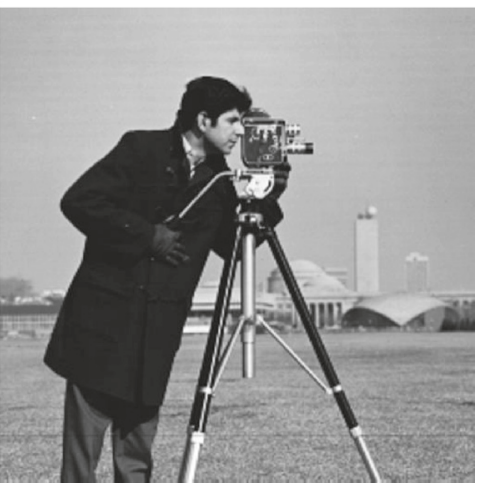

(d) Cameraman $(q=140)$

Fig. 8 Watermarked images for $k=8$. a Watermarked image of Lena ( $q=55)$. b Watermarked image of Jet ( $q=44)$. $\mathbf{c}$ Watermarked image of Peppers $(q=51)$. $\mathbf{d}$ Watermarked image of Cameraman $(q=50)$

$$
\xi^{*}=\frac{2}{255^{2} m n}\left(\mathrm{WW}^{\mathbf{T}}\right)^{-1}\left(\mathrm{~W} \hat{\lambda}_{D^{k}-y_{1}}\right)
$$

Replacing Eq. 32 with Eq. 29a yields the optimal embedded singular values

$$
\begin{aligned}
\hat{\lambda}_{D^{k}}{ }^{*} & =\lambda_{D^{k}}-\frac{255^{2} m n}{2} \xi^{*} \mathbf{W}^{\mathbf{\top}} \\
& =\lambda_{D^{k}}-\mathrm{W}^{\mathbf{\top}}\left(\mathrm{W} \mathbf{W}^{\mathbf{\top}}\right)^{-1}\left(\mathrm{~W} \lambda_{D^{k}}-y_{1}\right)
\end{aligned}
$$

By using $y_{2}$ instead of $y_{1}$ yields the optimal embedded singular values

$$
\hat{\lambda}_{D^{k}}{ }^{*}=\lambda_{D^{k}}-\mathrm{W}^{\mathbf{T}}\left(\mathrm{W} \mathbf{W}^{\mathbf{T}}\right)^{-1}\left(\mathrm{~W} \lambda_{D^{k}}-y_{2}\right)
$$

for the situation " $\mu_{j}{ }^{k}$ mode $2 \neq \beta_{i}$ and $\mu_{j}{ }^{k} \sum_{i=1}^{r} \lambda_{i}^{k} /_{q}=0$ ".

By using $y_{3}$ instead of $y_{1}$ yields the optimal embedded singular values

$$
\hat{\lambda}_{D^{k}}=\lambda_{D^{k}}-\mathrm{M}^{\mathbf{T}}\left(\mathrm{W} \mathrm{W}^{\mathbf{T}}\right)^{-1}\left(\mathrm{~W} \lambda_{D^{k}}-y_{3}\right)
$$

for the situation " $\mu_{j}{ }^{k}$ mode $2 \neq \beta_{i}$ and $\mu_{j}{ }^{k}-\left\lfloor\left.\sum_{i=1}^{r} \lambda_{i}^{k}\right|_{q}\right\rfloor \neq$ 0 ". Figure 4 shows the proposed optimal embedding process in detail.

\section{Experimental results}

This section presents experimental results that indicate the performance of the proposed image-watermarking scheme. Forty host images including the four images, Lena, Jet, Peppers, and Cameraman, each a size of $512 \times$ 512 , are decomposed into three levels by applying DWT; then, the watermark is embedded into the LH3 and HL3 coefficients. Figure 5 shows that the watermarked image can keep a high and stable PSNR (almost $54.5 \mathrm{~dB}$ ) even when the number of coefficients for embedding a watermark bit increases. This feature indicates the proposed optimization embedding formula using Lagrange principle. In order to compare with the SVD-based method [25], PSNR is fixed to be $55 \mathrm{~dB}$. Table 1 shows the comparison

Table 2 JPEG compression

\begin{tabular}{lllll}
\hline Quality factor & & 10 & 20 & 30 \\
\hline Reference [24] & & 15.08 & 8.67 & 3.92 \\
Reference [25] & & 13.22 & 9.12 & 4.03 \\
Reference [27] & 11.07 & 8.16 & 3.53 \\
Proposed method & $k=r=2$ & 15.77 & 9.32 & 5.46 \\
& $k=r=4$ & 12.68 & 8.13 & 3.12 \\
& $k=r=8$ & 11.15 & 4.84 & 0.97 \\
\hline
\end{tabular}


Table 3 JPEG2000 compression

\begin{tabular}{lllll}
\hline Quality factor & & 10 & 20 & 30 \\
\hline Reference [24] & & 16.36 & 9.88 & 3.42 \\
Reference [25] & & 14.25 & 8.62 & 4.27 \\
Reference [27] & & 15.18 & 9.13 & 3.58 \\
Proposed method & $k=r=2$ & 13.25 & 7.08 & 4.96 \\
& $k=r=4$ & 13.16 & 6.73 & 3.42 \\
& $k=r=8$ & 13.22 & 5.13 & 1.12 \\
\hline
\end{tabular}

of the embedding capacity under fixed PSNR $=55$. Figure 6 shows the original images, and Figs. 7 and 8 show the watermarked images obtained with different parameters.

To evaluate the robustness of the proposed method, the 40 images including the four images, Lena, Jet, Peppers, Cameraman, are tested. After the embedding process, four attacks are adopted to test the robustness of the embedded watermark in cases $k=4$ and $k=8$. The robustness is measured by BER defined by

$$
\mathrm{BER}=\frac{B_{\text {error }}}{B_{\text {total }}} \times 100 \%
$$

where $B_{\text {error }}$ and $B_{\text {total }}$ denote the number of error bits and the number of total bits, respectively. The method proposed herein is compared with the SVDbased method using the spread-spectrum technique in the DWT domain [25]. The test of robustness supports the following conclusions:

(1)JPEG compression and JPEG2000 compression are the most popular compression methods. They are widely used to reduce the sizes of images. Usually, an image is compressed before it is transmitted over the Internet. Table 2 and Table 3 concern the compression of the 40 watermarked images by JPEG compression and JPEG2000 compression with different quality factors. The average BER of the proposed method is much lower than the other methods in cases $k=r=4$ and $k=r=8$. At the same time, the average BER of the proposed method decreases as the parameter $k$ increases.

Table 4 Gaussian noise $($ mean $=0$ )

\begin{tabular}{lrrrr}
\hline \multicolumn{1}{l}{ Method and parameter } & & & \\
\hline & & 0.0002 & 0.0005 & 0.0009 \\
\hline Reference [24] & & 24.26 & 38.51 & 42.63 \\
Reference [25] & & 19.92 & 33.20 & 38.28 \\
Reference [27] & & 20.28 & 37.12 & 38.98 \\
Proposed method & $k=r=2$ & 7.13 & 21.73 & 34.96 \\
& $k=r=4$ & 6.69 & 21.87 & 25.58 \\
& $k=r=8$ & 5.46 & 13.28 & 22.65 \\
\hline
\end{tabular}

Table 5 Median filter

\begin{tabular}{lllll}
\hline & & 3 & 4 & 5 \\
\hline Reference [24] & & 29.06 & 33.91 & 32.03 \\
Reference [25] & & 29.41 & 32.61 & 32.18 \\
Reference [27] & & 29.08 & 30.14 & 30.27 \\
Proposed method & $k=r=2$ & 5.71 & 24.21 & 21.58 \\
& $k=r=4$ & 5.27 & 27.73 & 29.68 \\
& $k=r=8$ & 8.59 & 35.15 & 48.43 \\
\hline
\end{tabular}

(2) Table 4 shows the robustness against Gaussian noise with different means and variances. By testing the 40 watermarked images, the average BER of the proposed method is lower than other methods. As the parameter $k$ increases, the average BER of the proposed method decreases.

(3) Table 5 shows the robustness against median filtering with different radii in pixels. By testing the 40 watermarked images, the average BER of the proposed method is still much lower than other methods in cases $k=r=2$ and $k=r=4$. As the parameter $k$ increases, the average BER of the proposed method also increases a little.

(4) Table 6 shows the performance against rotation attack with different degrees. By testing the 40 watermarked images, the average BER of the proposed method is slightly higher than other methods.

From the above discussion, the proposed method performs better than the method in [25] except for the rotation attack. And as the parameter $k$ increases, the BER decreases as well except for the JPEG 2000 compression. To conclude, the proposed method is acceptable for its better performance except for the rotation attack.

\section{Conclusions}

This study improved the robustness of traditional SVDbased image watermarking by using optimization-based quantization on multiple singular values in the wavelet domain. Experimental results show that the watermarked

Table 6 Rotation

\begin{tabular}{lllll}
\hline Degree & & & & \\
\hline & & 1 & 4 \\
\hline Reference [24] & & 22.61 & 26.35 & 27.56 \\
Reference [25] & & 17.11 & 23.37 & 23.16 \\
Reference [27] & & 19.24 & 23.67 & 25.13 \\
Proposed method & $k=r=2$ & 38.57 & 40.03 & 40.35 \\
& $k=r=4$ & 37.07 & 38.89 & 39.67 \\
& $k=r=8$ & 37.31 & 37.65 & 40.31 \\
\hline
\end{tabular}


image can keep a high PSNR and achieve a better BER even when the number of coefficients for embedding a watermark bit increases. In particular, the robustness against JPEG compression, Gaussian noise, and median filtering is significantly improved. The future work is the consideration of improving robustness against rotation.

\section{Acknowledgments}

This work is partially supported under the grand MOST 103-2115-029-003.

\section{Author details}

${ }^{1}$ Department of Applied Mathematics, Tunghai University, Taichung 407, Taiwan. ${ }^{2}$ Department of Industrial Education and Technology, National Changhua University of Education, Changhua 500, Taiwan. ${ }^{3}$ Department of Business Administration, Tunghai University, Taichung, Taiwan.

\section{Received: 8 February 2015 Accepted: 1 June 2015}

Published online: 05 August 2015

\section{References}

1. F Hartung, M Kutter, Multimedia watermarking techniques. Proceedings of the IEEE, 1999, p. 87

2. M Alghoniemy, AH Tewfik, Geometric distortion correction in image watermarking, in Proceedings SPIE Security and Watermarking of Multimedia Contents I/ 3971, 2000, pp. 82-89

3. M Alghoniemy, AH Tewfik, Progressive quantized projection watermarking scheme, in Proceedings $7^{\text {th }}$ ACM International Multimedia Conference, Orlando, FL, 1999, pp. 295-298

4. B Chen, GW Wornell, Quantization index modulation: a class of provably good methods for digital watermarking and information embedding. IEEE Trans. Inf. Theory 47, 1423-1443 (2001)

5. P Kumswat, K Attakitmongcol, A Striaew, A new approach for optimization in image watermarking using genetic algorithms. IEEE Trans. Signal Process. 53(12), 4707-4719 (2005)

6. MU Celik, G Sharma, AM Tekalp, E Saber, Lossless generalized-LSB data embedding. IEEE Trans. Image Process. 14(2), 253-266 (2005)

7. LC Lin, YB Lin, CM Wang, Hiding data in spatial domain images with distortion tolerance. Elsevier: Computer Standards and Interfaces 31 458-464 (2009)

8. R Liu, T Tan, An SVD-based watermarking scheme for protecting rightful ownership. IEEE Transactions on Multimedia 4(1), 121-128 (2002)

9. C-C Chang, P Tsai, C-C Lin, SVD-based digital image watermarking scheme. Pattern Recogn. Lett. 26(10), 1577-1586 (2005)

10. KL Chung, WN Yang, YH Huang, ST Wu, YC Hsu, On SVD-based watermarking algorithm. Application. Math. Comput. 188, 54-57 (2007)

11. RA Ghazy, NA El-fishawy, MM Hadhoud, MI Dessouky, FEA El-Samie, An efficient block-by-block SVD-based image watermarking scheme, in 2007 Radio Science Conference, Cairo, 2007, pp. 1-9

12. SF Lin, SC Shie, JY Guo, Improving the robustness of DCT-based image watermarking again JPEG compression. Elsevier: Computer Standards and Interfaces 32, 57-60 (2010)

13. CC Lin, PF Shiu, High capacity data hiding scheme for DCT-based images. J. Inform. Hiding. Multimedia. Signal. Process. 1(3), 220-240 (2010)

14. H Qaheri, A Mustafi, S Banerjee, Digital watermarking using ant colony optimization in fractional fourier domain. J. Inform. Hiding. Multimedia. Signal. Process. 1(3), 179-189 (2010)

15. CH Manuel, GU Francisco, NM Mariko, HM Pérez-Meana, Robust hybrid color image watermarking method based on DFT domain and 2D histogram modification. Springer: Signal Image and Video Processing 8(1), 49-63 (2014)

16. L Xiao, H Wu, Z Wei, Multiple digital watermarks embedding in wavelet domain with multiple-based number. J. Computer. Aided. Design. Computer. Graphics. 15(2), 200-204 (2003)

17. $\mathrm{P}$ Bao, $X \mathrm{Ma}$, Image adaptive watermarking using wavelet domain singular value decomposition. IEEE Transactions on Circuits and Systems for Video Technology 15(1), 96-102 (2005)

18. E Ganic, AM Eskicioglu, Robust embedding of visual watermarks using DWT-SVD. J. Electronic. Imaging. 14(4), 1-13 (2005)
19. M Sharkas, B Youssef, $N$ Hamdy, An adaptive image-watermarking algorithm employing the DWT. the $23^{\text {th }}$ National Radio Science Conference, 2006, pp. 14-16

20. CT Li, Reversible watermarking scheme with image-independent embedding capacity. IEEE Proceedings on Vision, Image, and Signal Processing 152(6), 779-786 (2006)

21. CV Serdean, MK Ibrahim, A Moemeni, MM Al-Akaidi, Wavelet and multiwavelet watermarking. IET Image Process. 1(2), 223-230 (2007)

22. OZ Azza, M Achraf, B Ammar, Wavelet domain watermark embedding strategy using TTCQ quantization. J. Computer Sci. Network Security. 7(6), 165-170 (2007)

23. N Li, X Zheng, Y Zhao, H Wu, S Li, Robust algorithm of digital image watermarking based on discrete wavelet transform. International Symposium on Electronic Commerce and Security, 2008

24. B Gaurav, R Balasubramanian, A new robust reference watermarking scheme based on DWT-SVD. Elsevier: Computer Standards and Interfaces, 2009, pp. 1-12

25. CC Lai, CC Tsai, Digital image watermarking using discrete wavelet transform and singular value decomposition. IEEE Trans. Instrum. Meas. 59(11), 3060-3063 (2010)

26. S-T Chen, H-N Huang, C-Y Hsu, Optimization-based image watermarking scheme in the wavelet-domain, in 2010 Fourth International Conference on Genetic Evolutionary Computing, ShenZhen, China, 2010, pp. 671-674

27. K Loukhaoukha, JY Chouinard, MH Taieb, Optimal image watermarking algorithm based on LWT-SVD via multi-objective and colony optimization. J. Inform. Multimedia. Signal. Process. 2(4), 303-319 (2011)

28. A Mishra, C Agarwal, A Sharma, P Bedi, Optimized gray-scale image watermarking using DWT- SVD and firefly algorithm. Elsevier: Expert Systems with Applications 41, 7858-7867 (2014)

29. S-T Chen, H-N Huang, W-M Kung, C-Y Hsu, Optimization-based image watermarking with integrated quantization embedding in the wavelet domain. Springer: Multimedia Tools and Applications, 2015. doi:10.1007/s11042-015-2522-8

30. S-T Chen, G-D Wu, H-N Huang, Wavelet-domain audio watermarking scheme using optimisation-based quantisation. IET Proceedings on Signal Processing 4(6), 720-727 (2010)

31. S-T Chen, H-N Huang, C-C Chen, K-K Tseng, S-Y Tu, Adaptive audio watermarking via the optimization point of view on wavelet-based entropy. Elsevier: Digital Signal Processing, 2013, pp. 971-980

\section{Submit your manuscript to a SpringerOpen ${ }^{\odot}$ journal and benefit from:}

- Convenient online submission

Rigorous peer review

- Immediate publication on acceptance

- Open access: articles freely available online

- High visibility within the field

- Retaining the copyright to your article

Submit your next manuscript at $>$ springeropen.com 